\title{
Frequency thresholds for two-flash flicker and critical flicker: Why they differ
}

\author{
ROBERT M. HERRICK \\ Naval Air Development Center, Warminster, Pennsylvania 18974
}

\begin{abstract}
The minimum interval detectable between two successive flashes is longer than the minimum interval detectable when many flashes are viewed. The probability summation hypothesis is suggested to explain the difference. For a given flash duration, the minimum interval detectable decreases as a function of the number of flashes, $n$, until $n$ equals about 8 or 10; further increases in $n$ cause no further changes in this interval. Of the total decrease in the interval, from $n=2$ to $n=99$, about $90 \%$ is explained by the probability summation hypothesis.
\end{abstract}

The minimum interval detectable between successive flashes is much longer when two flashes are viewed than when many flashes are viewed. For example, with constant-luminance flashes of $0.03 \mathrm{msec}$, King (1962) found that, for the detection of inhomogeneity in the presentation, two flashes required an interval of $58.1 \mathrm{msec}$, while, with many flashes, the threshold interval between successive flashes was only $24.9 \mathrm{msec}$. With two flashes, the experiment is called the two-flash-flicker (TFF) experiment; with many flashes, the term customarily used is critical flicker frequency (CFF). ${ }^{1}$

Typically, the results of TFF and CFF experiments are summarized by a frequency measure. With the flash duration, $t$, and the threshold interval between successive flashes, $i$, measured in milliseconds, the frequency in hertz is given by the equation: frequency = $1,000 /(\mathrm{t}+\mathrm{i})$. Thus, in King's experiment, the threshold frequency with two flashes is $1,000 /(0.03+58.1)$, or $17.2 \mathrm{~Hz}$, and the threshold frequency with many flashes is $40.1 \mathrm{~Hz}$. Specified in frequency, several studies [see Brown (1965) for a review of the studies] show CFF to be about two or three times TFF. The present article suggests why CFF and TFF differ.

\section{ANALYSIS OF THE PROBLEM}

The probability summation hypothesis is an explanatory concept that has been invoked to explain a variety of visual data (e.g., see Pirenne, 1943; Bouman \& Van den Brink, 1952; Uetsuki \& Ikeda, 1970; Herrick, 1973a). The hypothesis will be used here as an explanation of the difference between TFF and CFF.

First, consider how the probability summation hypothesis applies to the present problem. Say, two 1 -msec flashes are presented to an $O$, who responds "Yes" if he detects the interval between the two flashes and "No" if the two flashes appear as one. Similarly, three $1-\mathrm{msec}$ flashes are presented, and the $O$ responds "Yes" if he detects any inhomogeneity in the presentation and "No" if the three flashes are perceived as one. And, in general, if any number of flashes are presented, $O$ responds "Yes" if one or more of the intervals are detected; otherwise, he responds "No."

When two flashes (with a given interval) are presented, the probability of a "yes" is $p$ and the probability of a "no" is $q$, where $q=1-p$. When three like flashes, similarly separated, are presented, O will say "Yes" if he detects either the first interval or the second, or both. Only if he detects neither interval will he say "No." Thus, considering the intervals as independent events in a probability sense, with two intervals the probability of a "no" equals the probability of not detecting the first interval times the probability of not detecting the second interval, namely, $\mathrm{q} \times \mathrm{q}$, or $\mathrm{q}^{2}$; thus, the probability of a "yes" is $1-q^{2}$. For example, if $p$ is 0.2929 for a given interval, the probability of a "yes" when two like intervals are presented is $1-(1-0.2929)^{2}$, or 0.500 .

Generalizing from the above development indicates that

$$
P_{n}=i-q^{n}=1-(1-p)^{n},
$$

where $p$ is the probability of a "yes" when one interval is presented, $n$ is the number of intervals presented, and $P_{n}$ is the probability of a "yes" when $n$ intervals are presented.

Given $\mathrm{p}$ and $\mathrm{n}, \mathrm{P}_{\mathrm{n}}$ can be computed by Eq. 1 . Conversely, given $P_{n}$ and $n, p$ can be computed. Thus, if three $1-\mathrm{msec}$ flashes separated by intervals of $39.0 \mathrm{msec}$ yield a $P_{n}=0.500$, then two 1 -msec flashes separated by $39.0 \mathrm{msec}$ should yield a $p=0.2929$. Similarly. if $\mathbf{P}_{\mathbf{n}}=$ 0.500 with four 1 -msec flashes separated by intervals of $34.5 \mathrm{msec}, \mathrm{p}$ should equal 0.2063 with two $1-\mathrm{msec}$ flashes separated by an interval of $34.5 \mathrm{msec}$. Derived in this manner, the set of $p$ values, $0.2929,0.2063$, etc., with their associated intervals, $39.0,34.5 \mathrm{msec}$, etc.. describe a psychometric function. i.e.. a function showing the probability of a "yes," $p$, as a function of the interval between the two flashes. If such a psychometric function can be derived from sets of values of $P_{n}, n$, and the associated interval, the data are described by the probability summation hypothesis. 


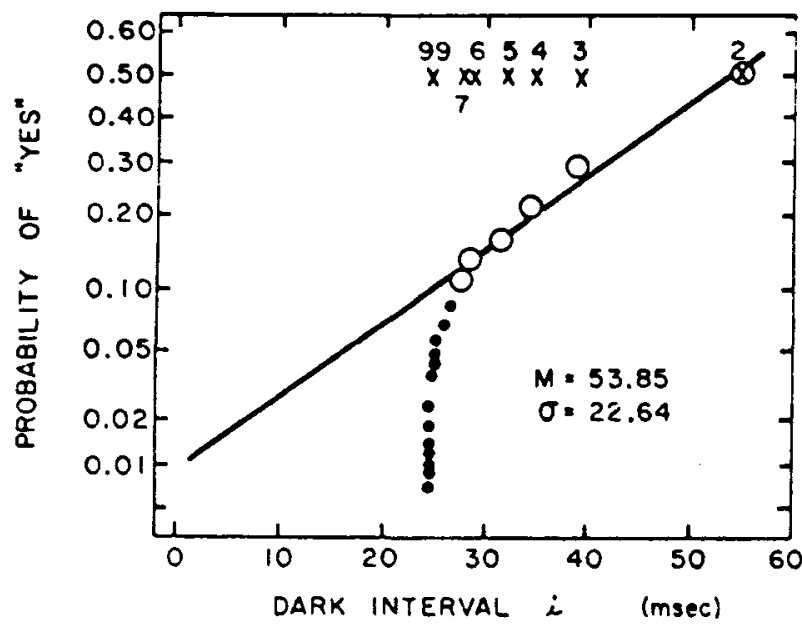

Fig. 1. Probability of a "yes" as a function of the interval separating successive $1-\mathrm{msec}$ flashes. Each $X$ gives the threshold dark interval found by Mahneke (1958) and plotted as a $p=0.50$ value. The number next to an $X$ gives the number of flashes. The circles $(O$ and $\bullet)$, derived from the probability summation hypothesis, give the probability of a "yes" for two flashes (one interval). The vertical scale is a cumulative normal scale. The line is a least-squares fit to the $O$ points.

\section{ANALYSIS OF EXPERIMENTAL DATA}

The analysis given above can be tested with experimental data. The data to be used were read from Fig. 1b of Mahneke's (1958) paper. Briefly, Mahneke's procedure was as follows: "The observer is ... exposed to two successive light flashes, and the duration of the dark interval which just permits fusion is determined. This is repeated for 17 different numbers of successive light flashes $(3,4,5,6$, etc.) and six different flash durations $(1,2,5,10,20$, and $50 \mathrm{msec})$ [p. 13]." In the analysis that follows, the flicker interval, rather than the fusion interval, is required, so Mahneke's fusion interval will be considered to be a flicker interval.

The threshold obtained in a method of limits experiment is approximately equal to the stimulus that yields a probability of a "yes" of 0.500 in a constant stimuli experiment (Herrick, 1967, 1970a, 1970b, 1973b; Pollack, 1968). Thus, a threshold interval of Mahneke's experiment may be taken as the interval that corresponds to a $P_{n}=0.500$ in a constant stimuli experiment.

First, consider Mahneke's data for the cases when the flash duration, $t$, is 1 msec. With two flashes, the threshold interval found experimentally was $55.0 \mathrm{msec}$ (see Fig. 1). Thus, with one interval, the probability of a "yes" is 0.500 when $i=55 \mathrm{msec}$. With three flashes (two intervals), the threshold interval was $39.0 \mathrm{msec}$. From the development of the preceding section, this means that when $\mathrm{i}=39.0 \mathrm{msec}$, the probability of a "yes" $\left(\mathrm{P}_{n}\right)$ for two intervals is 0.500 , and the probability of a "yes" (p) for one interval is 0.2929 . Thus, the derived point in Fig. 1 is plotted at an interval of $39.0 \mathrm{msec}$, with a $\mathrm{p}$ of
0.2929. In Fig. 1, the threshold intervals obtained experimentally are represented as Xs, and the derived $\mathrm{p}$ values associated with the threshold intervals are shown as circles. For the derived points based on cases of 2,3 , $4,5,6$, and 7 flashes, a straight line, fitted by the method of least squares, is given in Fig. 1. The straight line is the psychometric function for two flashes, i.e., for a single interval. The fit of the derived points to the straight line indicate that, from 2 to 7 flashes, the probability summation hypothesis adequately describes the data. Beyond 7 flashes, the derived points, shown in Fig. 1 as small circles, deviate from the straight line.

In Fig. 2, a similar analysis is given for a flash duration of $50 \mathrm{msec}$. In this case, the straight line gives a good description of the data up to 9 flashes. Analyses for flash durations of $2,5,10$, and $20 \mathrm{msec}$ were also performed, and the results, not shown, were similar to those of Figs. 1 and 2. For each flash duration, the psychometric function described the data down to the interval at which $\mathrm{p}$ equaled about 0.10 .

The process described above may be reversed: from a psychometric function for 2 flashes (1 interval), the threshold interval expected for 3 flashes, for 4 flashes, for 5 flashes, etc., can be calculated. Using the psychometric functions of Figs. 1 and 2, these calculations were made, and the results are presented as the curves in Fig. 3. The curves, of course, represent expectations based on the probability summation hypothesis. For the 1-msec flash duration in Fig. 3, the interval $i$, found experimentally, decreases from $55.0 \mathrm{msec}$ at 2 flashes to $24.5 \mathrm{msec}$ at 99 flashes. The curve describes the decrease from $55.0 \mathrm{msec}$ down to $28.5 \mathrm{msec}$. Thus, $87 \%$ of the decrease is described by the probability summation hypothesis. For the data where $t$

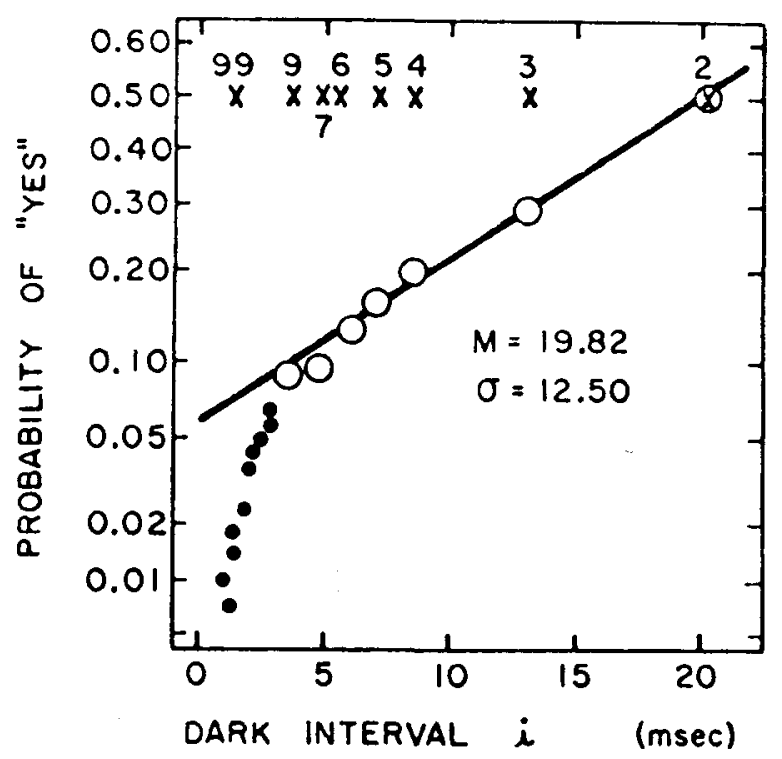

Fig. 2. Probability of a "yes" as a function of the interval separating successive 50 -msec flashes. Other notations are like those of Fig. 1. 
$=50 \mathrm{msec}, 95 \%$ of the decrease is described by the probability summation hypothesis.

With some flash durations, the total decrease in the interval, from 2 to 99 flashes, cannot be described by the hypothesis, because the psychometric function goes to an interval of $0.0 \mathrm{msec}$, as in Fig. 2. The Fig. 3 data for the 50-msec flashes illustrate the same point: at about 12 flashes, the curve reaches an interval of $0.0 \mathrm{msec}$. Obviously, an interval of zero cannot be detected. With other flash durations (see Fig. 3), the data points could follow the probability summation curve for a large number of flashes, but they do not. Rather, $O$ reacts in the same way to any number of flashes above 8 or 10 . No verifiable hypothesis for the deviation from the probability summation hypothesis is offered at this time. [It should be noted, however, that with a different visual problem, data were well described by the probability summation hypothesis up to, but not beyond, 12 "events" (Herrick 1973a).]

The data points of Fig. 3 can be used to calculate TFF and CFF. For example, for 2 flashes, $t=1 \mathrm{msec}$ and $\mathrm{i}=$ $55.0 \mathrm{msec}$; thus, frequency $=1,000 /(\mathrm{t}+\mathrm{i})=17.9 \mathrm{~Hz}$. With $\mathrm{t}=1 \mathrm{msec}$, for 99 flashes, which corresponds to CFF, frequency $=39.2 \mathrm{~Hz}$.

\section{TFF AND CFF WHEN FLASH DURATION EQUALS THE INTERVAL BETWEEN SUCCESSIVE FLASHES}

The analysis given above describes the difference between TFF and CFF when the flash duration, $t$, is held constant and the interval, $i$, is varied. The most common experimental procedure, however, is to set $t$

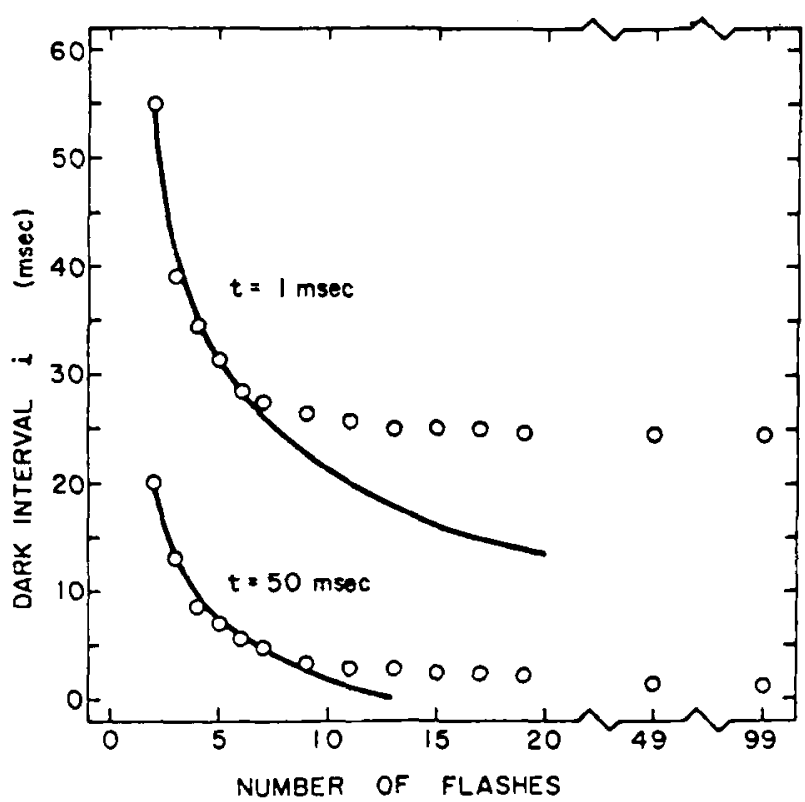

Fig. 3. Threshold dark interval as a function of the number of flashes, with flash durations of 1 and $50 \mathrm{msec}$. Data points are from Mahneke (1958): curves represent the probability summation hypothesis.

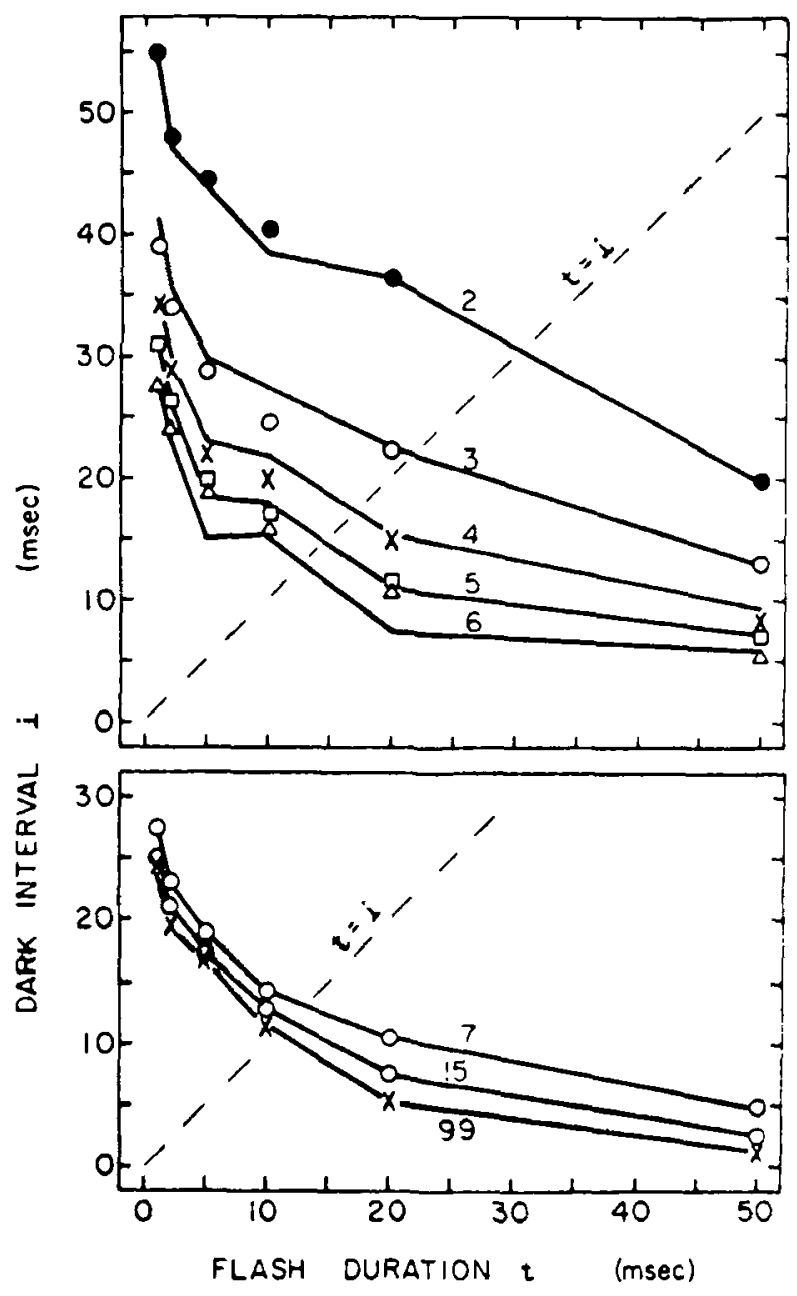

Fig. 4. Threshold dark interval as a function of flash duration. The parameter is the number of flashes. The lines in the upper panel were derived from the probability summation hypothesis.

equal to $i$, and then vary both $t$ and $i$ concomitantly. Unfortunately, such a procedure confounds the variables, because both $t$ and $i$ are relevant variables (see Fig. 3).

Nevertheless, the case of $t=i$ may be analyzed with the help of Mahneke's data. Plots of $t$ vs $i$ for $n$ flashes can be derived from Fig. 3. For example, for 2 flashes. if $\mathrm{t}=1 \mathrm{msec}, \mathrm{i}=55.0 \mathrm{msec}$, and if $\mathrm{t}=50 \mathrm{msec}$. $\mathrm{i}=$ $20.0 \mathrm{msec}$. (The other sets of Mahneke's data for $t=2$. 5,10 , and $20 \mathrm{msec}$ fall, in appropriate order. between the data for $t=1 \mathrm{msec}$ and $t=50 \mathrm{msec}$ in Fig. 3.) The experimental points of Mahneke, relating $t$ and $i$ for a given number of flashes, are given in Fig. 4. Because the different points associated with each function (e.g.. for ? flashes) were obtained in different sessions, the functions are not as smooth as those of Fig. 3. In the upper panel of Fig. 4, the lines associated with each function are based on probability summation functions like those of Fig. 3. Such lines in the upper panel of Fig. 4 are based on different psychometric functions. derived from data obtained in different sessions. They 


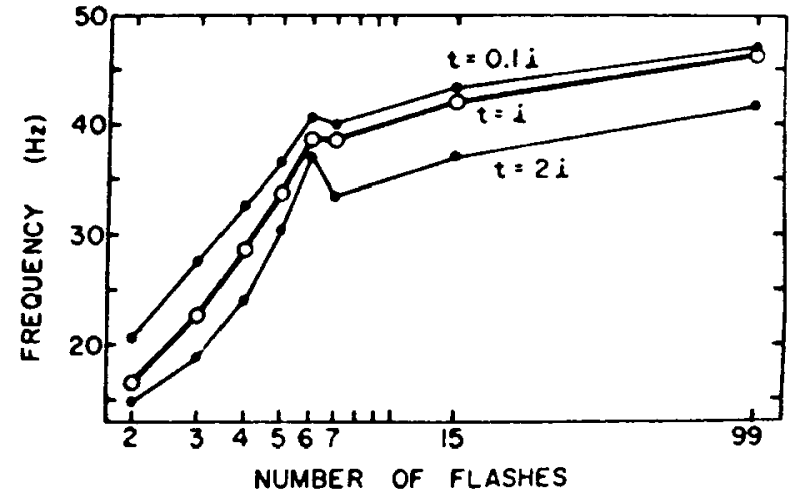

Fig. 5. Frequency as a function of the number of flashes on a $\log$ scale. Frequency $=1,000 /(t+i)$, with $t$ the flash duration and $i$ the interval between successive flashes. Middle curve, $t$ equals interval $i$; upper curve, $t$ is $1 / 10$ of $i$; lower curve, $t$ is twice i.

are adequate for our purposes, however. In the lower panel of Fig. 4, the experimental points are simply connected by straight lines, because the probability summation hypothesis is a poor fit when the number of flashes is large.

The dashed lines in Fig. 4 represent cases when the flash duration, $t$, equals the interval between flashes, $i$. From the intersection of the dashed line with the line for a given number of flashes, the value of $t=i$ may be read. For example, the dashed line for $\mathrm{t}=\mathrm{i}$ intersects the line for 2 flashes at $\mathrm{t}=\mathrm{i}=30.5 \mathrm{msec}$. Thus, with 2 flashes, $\mathrm{t}=\mathrm{i}=30.5 \mathrm{msec}$, and the frequency $=16.4 \mathrm{~Hz}$. By similar steps, the frequency was determined for 3,4 , $5,6,7,15$, and 99 flashes. The results are given in Fig. 5 by the curve labeled $t=i$. By analogous steps, to indicate the influence of different light-dark ratios, the frequencies were determined for 2 to 99 flashes when $t=$ $0.1 \mathrm{i}$ and when $t=2 i$, and the results are included in Fig. 5.

\section{CONCLUSION}

The argument presented in the foregoing analysis may be summarized in a few words. If the interval between successive, identical flashes is held constant, as the number of flashes increases from 2 , the probability of detecting one or more intervals increases. A mathematical statement of this idea is the probability summation hypothesis. The hypothesis gives a good description of experimental data for any number of flashes from 2 to about 8 or 10 . Any number of flashes greater than 8 or 10 is treated the same by 0 .

\section{REFERENCES}

Bouman, M. A., \& Van den Brink, G. On the integrate capacity in time and space of the human peripheral retina. Journal of the Optical Society of America, 1952, 42, 617-620.

Brown, J. L. Flicker and intermittent stimulation. In C. H. Graham (Ed.), Vision and visual perception. New York: Wiley, 1965. Pp. 251-320.

Herrick, R. M. Psychophysical methodology: Comparison of thresholds of the method of limits and of the method of constant stimuli. Perceptual \& Motor Skills, 1967, 24, 915-922.

Herrick, R. M. Psychophysical methodology: Deductions from the phi-gamma hypothesis and related hypotheses. Perception \& Psychophysics, 1970a, 7, 73-78.

Herrick, R. M. Psychophysical methodology. IV: Phi gamma hypothesis and the method of limits. Perception \& Psychophysics, 1970b, 8, 61-64.

Herrick, R. M. Foveal increment thresholds for multiple flashes. Journal of the Optical Society of America, 1973a, 63, 870-878.

Herrick, R. M. Psychophysical methodology. VI: Random method of limits. Perception \& Psychophysics, 1973b, 13, 548-554.

King, H. E. Two-flash and flicker fusion thresholds for normal and schizophrenic subjects. Perceptual \& Motor Skills, 1962, 14, 517-518.

Mahneke, A. Fusion threshold of the human eye as measured with two or several light flashes. Acta Ophthalmologica, 1958, 36, 12-18.

Pirenne, M. H. Binocular and uniocular threshold of vision. Nature, 1943, 152, 698-699.

Pollack, I. Computer simulation of threshold observations by method of limits. Perceptual \& Motor Skills, 1968, 26, 583-586.

Uetsuki, T., \& Ikeda, M. Study of temporal visual response by the summation index. Journal of the Optical Society of America, 1970, 60, 377-381.

\section{NOTE}

1. The term CFF is vaguer than TFF because CFF does not specify the number of flashes, although it is usually understood that a large number of flashes, 50 or 100 or more, are presented. On the other hand, CFF is more specific than TFF because CFF indicates that the threshold measurement is specified in frequency. Frequency, however, is a derived measure, based on the two basic measures, flash duration and the interval between flashes, and the frequency measurement may tend to obscure rather than clarify the relations among variables. Since most descriptions of data in the literature are given in frequency, the frequency measure, as well as the two basic measures, will be used in this report.

(Received for publication May 29, 1973; revision accepted August 22, 1973.) 